PHYSICAL REVIEW D 89, 071301(R) (2014)

\title{
Light sterile neutrino sensitivity at the nuSTORM facility
}

D. Adey, ${ }^{1}$ S. K. Agarwalla, ${ }^{2}$ C. M. Ankenbrandt,${ }^{3,{ }^{*}}$ R. Asfandiyarov, ${ }^{4}$ J. J. Back, ${ }^{5}$ G. Barker, ${ }^{5}$ E. Baussan, ${ }^{6}$ R. Bayes, ${ }^{7, \dagger}$ S. Bhadra, ${ }^{8}$ V. Blackmore, ${ }^{9}$ A. Blondel, ${ }^{4}$ S. A. Bogacz, ${ }^{10}$ C. Booth,${ }^{11}$ S. B. Boyd ${ }^{5}$ S. G. Bramsiepe, ${ }^{7}$ A. Bravar, ${ }^{4}$ S. J. Brice, ${ }^{1}$ A. D. Bross,${ }^{1}$ F. Cadoux,${ }^{4}$ H. Cease, ${ }^{1}$ A. Cervera, ${ }^{12}$ J. Cobb, ${ }^{9}$ D. Colling, ${ }^{13}$ P. Coloma,${ }^{14}$ L. Coney, ${ }^{15}$ A. Dobbs ${ }^{13}$ J. Dobson, ${ }^{13}$ A. Donini,${ }^{12}$ P. Dornan, ${ }^{13}$ M. Dracos, ${ }^{6}$ F. Dufour, ${ }^{4}$ R. Edgecock, ${ }^{16}$ M. Geelhoed, ${ }^{1}$ M. A. Uchida, ${ }^{13}$ T. Ghosh, ${ }^{12}$ J. J. Gómez-Cadenas, ${ }^{12}$ A. de Gouvêa, ${ }^{17}$ A. Haesler, ${ }^{4}$ G. Hanson, ${ }^{15}$ P. F. Harrison, ${ }^{5}$ M. Hartz ${ }^{8, \$}$ P. Hernández, ${ }^{12}$ J. A. Hernando Morata, ${ }^{18}$ P. Hodgson, ${ }^{11}$ P. Huber, ${ }^{14}$ A. Izmaylov, ${ }^{12}$ Y. Karadzhov, ${ }^{4}$ T. Kobilarcik, ${ }^{1}$ J. Kopp, ${ }^{19}$ L. Kormos ${ }^{20}$ A. Korzenev, ${ }^{4}$ Y. Kuno, ${ }^{21}$ A. Kurup, ${ }^{13}$ P. Kyberd, ${ }^{22}$ J. B. Lagrange, ${ }^{23}$ A. Laing, ${ }^{12}$ A. Liu, ${ }^{1,}$ J. M. Link, ${ }^{14}$ K. Long, ${ }^{13}$ K. Mahn ${ }^{24}$ C. Mariani, ${ }^{14}$ C. Martin, ${ }^{4}$ J. Martin, ${ }^{25}$ N. McCauley, ${ }^{26}$ K. T. McDonald, ${ }^{27}$ O. Mena, ${ }^{12}$ S. R. Mishra, ${ }^{28}$ N. Mokhov, ${ }_{1}^{1}$ J. Morfín, ${ }^{1}$ Y. Mori, ${ }^{23}$ W. Murray, ${ }^{16}$ D. Neuffer, ${ }^{1}$ R. Nichol, ${ }^{29}$ E. Noah, ${ }^{4}$ M. A. Palmer, ${ }^{1}$ S. Parke, ${ }^{1}$ S. Pascoli, ${ }^{30}$ J. Pasternak, ${ }^{13}$ R. Plunkett, ${ }^{1}$ M. Popovic, ${ }^{1}$ P. Ratoff, ${ }^{20}$ M. Ravonel, ${ }^{4}$ M. Rayner, ${ }^{4}$ S. Ricciardi ${ }_{11}^{16}$ C. Rogers, ${ }_{16}$ P. Rubinov ${ }^{1}$ E. Santos, ${ }^{13}$ A. Sato, ${ }^{21}$ T. Sen, ${ }^{1}$ E. Scantamburlo, ${ }^{4}$ J. K. Sedgbeer, ${ }^{13}$ D. R. Smith, ${ }^{22}$ P. J. Smith, ${ }^{11}$ J. T. Sobczyk, ${ }^{31}$ L. Søby, ${ }^{32}$ F. J. P. Soler, ${ }^{7}$ M. Sorel, ${ }^{12}$ P. Snopok, ${ }^{33, \|}$ P. Stamoulis, ${ }^{12}$ L. Stanco, ${ }^{34}$ S. Striganov, ${ }_{13}^{1}$ H. A. Tanaka, ${ }^{35}$ I. J. Taylor, ${ }^{5}$ C. Touramanis, ${ }^{26}$ C. D. Tunnell,,${ }^{9,}$ Y. Uchida ${ }^{13}$ N. Vassilopoulos, ${ }^{6}$ M. O. Wascko, ${ }^{13}$ A. Weber, ${ }^{9}$ M. J. Wilking, ${ }^{24}$ E. Wildner, ${ }^{32}$ and W. Winter ${ }^{36}$

(nuSTORM Collaboration)

\author{
${ }^{1}$ Fermi National Accelerator Laboratory, Box 500, Batavia, Illinois 60510-5011, USA \\ ${ }^{2}$ Institute of Physics, Sachivalaya Marg, Sainik School Post, Bhubaneswar 751005, Orissa, India \\ ${ }^{3}$ Muons Incorporated, 552 N. Batavia Avenue, Batavia, Illinois 60510, USA \\ ${ }^{4}$ University de Geneve, 24, Quai Ernest-Ansermet, 1211 Geneva 4, Switzerland \\ ${ }^{5}$ Department of Physics, University of Warwick, Coventry CV4 7AL, United Kingdom \\ ${ }^{6}$ IPHC, Université de Strasbourg, CNRS/IN2P3, F-67037 Strasbourg, France \\ ${ }^{7}$ School of Physics and Astronomy, Kelvin Building, University of Glasgow, \\ Glasgow G12 8QQ, Scotland, United Kingdom \\ ${ }^{8}$ Department of Physics and Astronomy, York University, \\ 4700 Keele Street, Toronto, Ontario M3J 1P3, Canada \\ ${ }^{9}$ Oxford University, Subdepartment of Particle Physics, Oxford, OX1 3RH United Kingdom \\ ${ }^{10}$ Thomas Jefferson National Accelerator Facility, Newport News, Virginia 23606, USA \\ ${ }^{11}$ University of Sheffield, Department of Physics and Astronomy, Hicks Building, Sheffield S3 7RH, \\ United Kingdom \\ ${ }^{12}$ Instituto de Física Corpuscular (IFIC), Centro Mixto CSIC-UVEG, Edificio Institutos Investigación, \\ Paterna, Apartado 22085, 46071 Valencia, Spain \\ ${ }^{13}$ Physics Department, Blackett Laboratory, Imperial College London, Exhibition Road, London SW7 $2 A Z$, \\ United Kingdom \\ ${ }^{14}$ Center for Neutrino Physics, Virginia Polytechnic Institute and State University, \\ Blacksburg, Virginia 24061-0435, USA \\ ${ }^{15}$ University of California, Riverside, California 92521, USA \\ ${ }^{16}$ STFC Rutherford Appleton Laboratory, Chilton, Didcot, Oxfordshire OX11 OQX, United Kingdom \\ ${ }^{17}$ Northwestern University, Evanston, Illinois 60208-3112, USA \\ ${ }^{18}$ Universidade de Santiago de Compostela (USC), \\ Departamento de Fisica de Particulas, E-15706 Santiago de Compostela, Spain \\ ${ }^{19}$ Max-Planck-Institut für Kernphysik, PO Box 103980, 69029 Heidelberg, Germany \\ ${ }^{20}$ Physics Department, Lancaster University, Lancaster LA1 4YB, United Kingdom \\ ${ }^{21}$ Osaka University, Osaka 560-0043, Japan \\ ${ }^{22}$ Centre for Sensors and Instrumentation, School of Engineering and Design, \\ Brunel University, Uxbridge, Middlesex UB8 3PH, United Kingdom \\ ${ }^{23}$ Kyoto University, Kyoto 606-8502, Japan \\ ${ }^{24}$ TRIUMF, 4004 Wesbrook Mall, Vancouver, British Columbia V6T 2A3, Canada \\ ${ }^{25}$ Department of Physics, University of Toronto, \\ 60 Saint George Street, Toronto, Ontario M5S 1A7, Canada \\ ${ }^{26}$ Department of Physics, Oliver Lodge Laboratory, University of Liverpool, \\ Liverpool L69 7ZE, United Kingdom \\ ${ }^{27}$ Princeton University, Princeton, New Jersey 08544, USA \\ ${ }^{28}$ Department of Physics and Astronomy, University of South Carolina, \\ Columbia, South Carolina 29208, USA
}




\author{
${ }^{29}$ Department of Physics and Astronomy, University College London, \\ Gower Street, London WC1E 6BT, United Kingdom \\ ${ }^{30}$ Institute for Particle Physics Phenomenology, Department of Physics, Durham University, \\ Durham DH1 3LE, United Kingdom \\ ${ }^{31}$ Institute of Theoretical Physics, University of Wroclaw, pl. M. Borna 9, 50-204 Wroclaw, Poland \\ ${ }^{32}$ CERN, CH-1211 Geneva 23, Switzerland \\ ${ }^{33}$ Illinois Institute of Technology, Chicago, Illinois 60616, USA \\ ${ }^{34}$ INFN, Sezione di Padova, 35131 Padova, Italy \\ ${ }^{35}$ Department of Physics and Astronomy, Hennings Building, The University of British Columbia, \\ 6224 Agricultural Road, Vancouver, British Columbia V6T 1Z1, Canada \\ ${ }^{36}$ Fakultät für Physik und Astronomie, Universität Würzburg Am Hubland, 97074 Würzburg, Germany
} (Received 27 August 2013; published 9 April 2014)

A facility that can deliver beams of electron and muon neutrinos from the decay of a stored muon beam has the potential to unambiguously resolve the issue of the evidence for light sterile neutrinos that arises in short-baseline neutrino oscillation experiments and from estimates of the effective number of neutrino flavors from fits to cosmological data. In this paper, we show that the nuSTORM facility, with stored muons of $3.8 \mathrm{GeV} / \mathrm{c} \pm 10 \%$, will be able to carry out a conclusive muon neutrino appearance search for sterile neutrinos and test the LSND and MiniBooNE experimental signals with $10 \sigma$ sensitivity, even assuming conservative estimates for the systematic uncertainties. This experiment would add greatly to our knowledge of the contribution of light sterile neutrinos to the number of effective neutrino flavors from the abundance of primordial helium production and from constraints on neutrino energy density from the cosmic microwave background. The appearance search is complemented by a simultaneous muon neutrino disappearance analysis that will facilitate tests of various sterile neutrino models.

DOI: 10.1103/PhysRevD.89.071301

PACS numbers: 14.60.St, 14.60.Ef, 14.60.Pq
The issue of light sterile neutrinos is one of general interest to particle physicists and cosmologists. Intriguing evidence from terrestrial neutrino sources exists for neutrino mixing between the three active neutrinos and light sterile neutrino species. Short-baseline neutrino oscillations observed by the LSND [1] and MiniBooNE [2] experiments, the collective evidence of the reactor neutrino anomaly [3] and the gallium anomaly [4-8] all point towards sterile neutrinos with masses at the electronvolt level. While these results are tantalizing, they are not conclusive on their own and there is tension with the disappearance searches, which exclude the best-fit light neutrino $[9,10]$. Furthermore, estimates of the effective number of neutrino flavors $[11,12]$ from fits to cosmological data suggest that this number is greater than three. These results are based on primordial helium production during big-bang nucleosynthesis and constraints on neutrino energy density from the cosmic microwave background. Assumptions based on the partial thermalization of USA.

*Also at Fermilab, P.O. Box 500, Batavia, IL 60510-5011,

Corresponding author.

Ryan.Bayes@glasgow.ac.uk

${ }^{\ddagger}$ Also at Department of Physics, University of Toronto, 60 St. George Street, Toronto, Ontario M5S 1A7, Canada.

${ }^{\S}$ Also at Indiana University Bloomington, 107S Indiana Ave, Bloomington, IN 47405, USA.

"Also at Fermi National Accelerator Laboratory, Box 500, Batavia, IL 60510-5011, USA.

"Also at NikHEF, Amsterdam, The Netherlands. the primordial neutrino species [13] and the inclusion of uncertainties in the Hubble constant [14] can be used to accommodate all the available data. Therefore, there is great interest to resolve the issue of the existence of light sterile neutrinos, with implications for particle physics and cosmology.

New ideas have recently been proposed, based on carrying out oscillation experiments from isotope decayat-rest sources [15] and other accelerator, reactor and active source neutrino experiments [16]. In this article we show that the nuSTORM facility, providing neutrino beams from the decay of muons in a storage ring, can unambiguously resolve the problem of the existence of light sterile neutrinos by providing a source for all short-baseline oscillation modes. This idea has evolved from previous neutrino factory work carried out in the context of sterile neutrino $(2+2)$ and $(3+1)$ models [17-19]. We will show in this article that the currently proposed nuSTORM accelerator facility is feasible, without the need for new technology, and that the analysis presented is realistic, in terms of the detector performance. The best sensitivity to sterile neutrinos can be achieved with the $\nu_{e} \rightarrow \nu_{\mu}$ oscillation channel, conjugate to the LSND measurement, but the simultaneous access to disappearance modes can be used to test the consistency of the neutrino oscillation hypothesis for the first time in a single experiment.

Muon decays in flight yield a neutrino beam with a precisely known flavor content and energy spectrum. The primary decay mode, $\mu^{+} \rightarrow e^{+} \nu_{e} \bar{\nu}_{\mu}$, is $98.6 \%$ of all muon decays. The remainder is made up of radiative decays, 


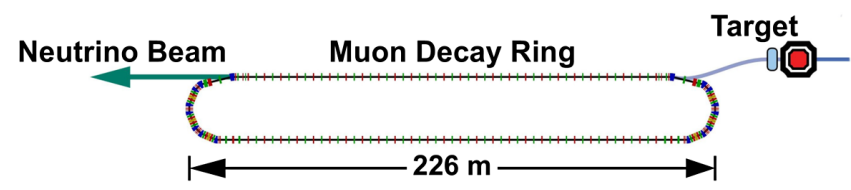

FIG. 1 (color online). A schematic of the storage ring configuration. Pions are injected into a straight section and must decay into muons before the first bend or be ejected from the ring. Muons that decay in the injection straight during subsequent turns produce the neutrino beam.

$\mu^{+} \rightarrow e^{+} \nu_{e} \bar{\nu}_{\mu} \gamma \quad($ B.R. $\approx 1.4 \%), \quad$ and $\mu^{+} \rightarrow e^{+} e^{-} e^{+} \nu_{e} \bar{\nu}_{\mu}$ (B.R. $\left.=(3.4 \pm 0.4) \times 10^{-5}\right)$ [20]. These decays all have the same neutrino content $\left(50 \% \nu_{e}, 50 \% \bar{\nu}_{\mu}\right)$, so any difference in the neutrino flavor would represent new physics. The energy spectrum of the muon decay positron has been measured to be consistent with the standard model at the level of a few parts in $10^{4}$ [21].

The nuSTORM facility has been designed to inject $5 \mathrm{GeV}$ pions into a muon storage ring [22], with a beam lattice in a race track configuration (Fig. 1). The effective straight for neutrino production is $185 \mathrm{~m}$ long and includes pion injection and extraction sections. The total circumference of the ring is $480 \mathrm{~m}$. The storage ring circulates muons with a central momentum of $3.8 \mathrm{GeV} / \mathrm{c}$ and has a momentum acceptance of $\approx \pm 10 \%$. Pions that do not decay prior to the first bend and muons produced from pion decay in the forward direction are removed by an extraction section at the end of the straight. Since the muons circulate many times between pion fills, neutrinos from pion decay are separated from the sample of neutrinos purely from muon decays through the use of a time cut that isolates decays immediately after injection. Muons that decay in the bends or in the opposing straight do not produce useful neutrinos. It is expected that $\approx 2 \times 10^{18}$ useful muon decays in the production straight that points toward the far detector site can be generated by nuSTORM from a total of $10^{21}$ protons on target (POT) over a total of ten years [22,23]. The neutrino beam has a dispersion of $29 \mathrm{mrad}$ from the boost of the muon decay and 4 mrad muon beam divergence in the production straight. The uncertainty in the neutrino flux is expected to be less than $0.5 \%$, due to the measurements to be carried out by beam monitoring devices in the decay run and at a near detector.

The well-defined neutrino beams available at the nuSTORM facility grant unparalleled opportunities for neutrino physics. Rates of accessible neutrino oscillation channels for stored $\mu^{+}$, assuming a simple $(3+1)$ sterile neutrino model [24] consistent with the LSND anomaly, are shown in Table I for $10^{21}$ POT. The probability of observing a $\nu_{e} \rightarrow \nu_{\mu}$ transition is given by

$$
P_{e \mu}=\sin ^{2} 2 \theta_{e \mu} \sin ^{2}\left(\frac{\Delta m^{2} L}{4 E}\right)
$$

TABLE I. Expected rates for neutrino oscillation channels observed at a $1.3 \mathrm{kt}$ detector, $2 \mathrm{~km}$ away from a muon storage ring with an exposure of $10^{21}$ POT.

\begin{tabular}{llrr}
\hline \hline Channel & Oscillation & \multicolumn{1}{c}{$N_{\text {osc }}$} & \multicolumn{1}{c}{$N_{\text {null }}$} \\
\hline$\nu_{\mu}$ Appearance & $\nu_{e} \rightarrow \nu_{\mu}$ CC & 332 & 0 \\
$\bar{\nu}_{\mu}$ Disappearance & $\bar{\nu}_{\mu} \rightarrow \bar{\nu}_{\mu}$ CC & 122322 & 128433 \\
$\nu_{e}$ Disappearance & $\nu_{e} \rightarrow \nu_{e}$ CC & 216657 & 230766 \\
NC Disappearance & $\bar{\nu}_{\mu} \rightarrow \bar{\nu}_{\mu} \mathrm{NC}$ & 47679 & 50073 \\
NC Disappearance & $\nu_{e} \rightarrow \nu_{e} \mathrm{NC}$ & 73941 & 78805 \\
\hline \hline
\end{tabular}

where $\theta_{e \mu}$ is an effective mixing angle, and $\Delta m^{2}$ is the effective mass difference, independent of the sterile neutrino model. In the $(3+1)$ model $\sin ^{2} 2 \theta_{e \mu} \equiv 4\left|U_{\mu 4}\right|^{2}\left|U_{e 4}\right|^{2}$ where $U_{e n}$ is an element of the Pontecorvo-MakiNakagawa-Sakata (PMNS) mixing matrix. Alternatively, the probability of observing a $\bar{\nu}_{\mu}$ disappearance transition is given by

$$
P_{\mu \mu}=1-\sin ^{2} 2 \theta_{\mu \mu} \sin ^{2}\left(\frac{\Delta m^{2} L}{4 E}\right),
$$

where $\sin ^{2} 2 \theta_{\mu \mu} \equiv 4\left|U_{\mu 4}\right|^{2}\left(1-\left|U_{\mu 4}\right|^{2}\right)$ in a $(3+1)$ model. A $\nu_{\mu}$ appearance experiment is conducted by observing $\mu^{-}$in the detector and a $\bar{\nu}_{\mu}$ disappearance experiment relies on identification of $\mu^{+}$in the detector. Therefore, the sensitivity to oscillations depends on the ability of the detector to distinguish the charge of the leptons produced in the neutrino charged current (CC) interactions. With the rates shown in Table I, a background acceptance of $10^{-4}$ is required for an appearance measurement. Direct measurement of the cross sections of both electron and muon neutrinos can be measured at a near detector site $50 \mathrm{~m}$ from the end of the decay straight. The number of $\nu_{e}$ and $\bar{\nu}_{\mu} \mathrm{CC}$ events (per 100 ton fiducial mass at the near detector) is $4.0 \times 10^{6}$ and $2.1 \times 10^{6}$, respectively, for a $10^{21}$ POT exposure. It is also possible to select $\mu^{-}$in the storage ring. This will yield a lower rate in the detection of appearance oscillations, and hence a reduced sensitivity, due to the difference in the cross section between neutrinos and antineutrinos $\left(1.8 \times 10^{6} \bar{\nu}_{e}\right.$ and $4.6 \times 10^{6} \nu_{\mu} \mathrm{CC}$ events would be observed in the near detector in this case).

A $1.3 \mathrm{kt}$ magnetized iron-scintillator calorimeter has been selected as the detector for short-baseline oscillation physics at nuSTORM, as it has excellent charge selection and detection characteristics for muons. This $6 \mathrm{~m}$ diameter detector is to be constructed of modules of $1.5 \mathrm{~cm}$ thick steel plates, and two layers of scintillator bars to yield $3 \mathrm{D}$ space points at each measurement plane. The overall length of this detector is $13 \mathrm{~m}$. Each scintillator bar has a cross section of $2.0 \times 0.75 \mathrm{~cm}^{2}$ and will be read out using silicon photomultipliers. For a schematic of this detector, see Fig. 2. The magnetic field will be generated by a $240 \mathrm{kA}$-turns current carried by 8 turns of a superconducting transmission line. This provides a toroidal magnetic field between 1.9 and $2.6 \mathrm{~T}$ within the steel. 


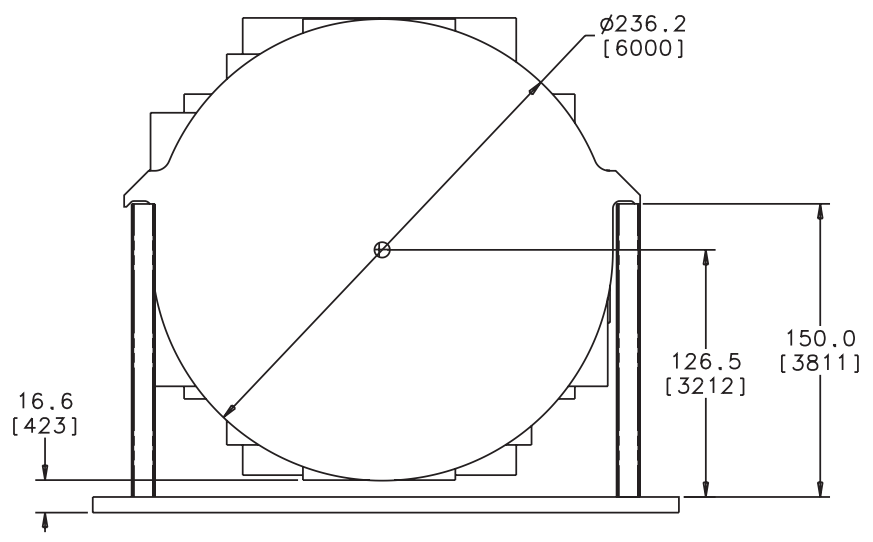

FIG. 2. A cross section of the prospective iron-scintillator neutrino detector $(6 \mathrm{~m}$ in diameter and $13 \mathrm{~m}$ in length).

A detailed simulation of the iron-scintillator far detector was developed from the neutrino factory far detector simulation [25]. This simulation uses the GENIE [26] package to simulate neutrino interactions in iron and scintillator, and GEANT4 [27] to simulate the interactions of the products with the detector material. A simple digitization is used to group ionization sites to particular paired scintillator bars and replicate the effects of resolution and attenuation within the scintillator bars. Tracks are reconstructed from the events through repeated application of a Kalman filter [28] to determine the momentum and charge of tracks. Multiple tracks are fit from each event and the longest track is defined as the muon. Other tracks, if present, are assumed to be the result of pion production and other particle shower processes. A track is reconstructed from pion or shower events in $1 \%$ of cases, necessitating further analysis to remove such events.

A multivariate analysis of reconstructed events is used to distinguish signal events from background with a high degree of purity. A series of cuts to perform a preselection of events were applied first. These are based on finding one or more tracks in the event; on successfully fitting the longest track; imposing a maximum momentum $p_{\mu}<$ $4 \mathrm{GeV} / \mathrm{c}$ for the longest track; applying a fiducial cut in which the longest track must start before the last $1 \mathrm{~m}$ of the detector; $60 \%$ of the hits assigned must be associated to the longest track; the relative error on the ratio $q / p$, where $q$ and $p$ are the fitted charge and momentum of the track, must satisfy $\sigma_{q / p} /(q / p)<10.0$; and the ratio of the initial curvature over the fitted curvature satisfies $\left(q_{\text {init }} / p_{\text {init }}\right) \times$ $(p / q)>0$. An approach was used based on the boosted decision tree (BDT) algorithm provided by the toolkit for mulitvariate analysis (TMVA) [29] subset of the ROOT [30] analysis package in which five track variables (shown in Table II) are used to discriminate between muons from $\nu_{\mu}$ $\mathrm{CC}$ interactions and all other types of interactions. The method reduces these five track variables to one classifier variable that runs between 0 and 1 , based on a training process that differentiates between $\nu_{\mu} \mathrm{CC}$ events, the
TABLE II. Variables used in the definition of the classifier for the multivariate analysis of events in the detector simulation.

\begin{tabular}{lc}
\hline \hline Variable & \multicolumn{1}{c}{ Description } \\
\hline Track quality & $\begin{array}{c}\sigma_{q / p} /(q / p) \text {, the normalized error } \\
\text { in the track curvature. } \\
\text { The number of sci. planes in track. } \\
\left(q_{\text {init }} / p_{\text {range }}\right) \times\left(p_{\text {fit }} / q_{\text {fit }}\right): \text { ratio of the } \\
\text { initial estimate and Kalman } \\
\text { Hits in trajectory } \\
\text { Curvature ratio }\end{array}$ \\
$\begin{array}{l}\text { Mean enentum. } \\
\text { Variation in energy }\end{array}$ & $\begin{array}{l}\sum_{i=0}^{N} \Delta E_{i} / N \text { for planes in track. } \\
\text { energy deposited per }\end{array}$ \\
& hit $\Delta E_{i}<\Delta E_{i+1}$. \\
\hline \hline
\end{tabular}

experimental signal, and $\bar{\nu}_{\mu}$ neutral current (NC) events, representing the experimental backgrounds. The trained multivariate analysis (MVA) is applied to simulations corresponding to the entries in Fig. 3(a) to determine the detector response to signal $(S)$ and background $(B)$ events. Given the expected number of oscillated and unoscillated neutrinos at the far detector, an optimal signal significance — quantified as $S / \sqrt{S+B}$ - is achieved for an appearance experiment when the classifier is restricted to values greater than 0.86 . This yields an integrated signal efficiency of 0.17 and a background efficiency of $4 \times 10^{-5}$. This background is predominantly due to charge misidentification from $\nu_{\mu} \mathrm{CC}$ events, but also contains pion decay and punch through from NC events. A cuts-based analysis was also studied [22,31], based on the number of hits in a trajectory and the track quality, but yielded a decreased physics sensitivity, with a signal efficiency of 0.16 and a background efficiency of $5 \times$ $10^{-5}$ at a higher energy threshold.

For a disappearance analysis, a different optimization is required since background rejection is a lesser concern. An optimization using a $\chi^{2}$ statistic between neutrino spectra, given the $(3+1)$ sterile neutrino hypothesis and the standard neutrino hypothesis, concludes that a neural network (MLPBNN) algorithm [29] that retains classifier values greater than 0.94 outperforms the BDT algorithm. The efficiency curves for the optimized analysis are shown in Fig. 3(b).

The detector response for each class of event shown in Fig. 3 is extracted from the detector simulation as a "migration" matrix of the probability of a neutrino generated in the $i$ th energy bin being reconstructed in the $j$ th energy bin. The migration matrices are input into a simulation of the oscillation experiment using the GLoBES software package [32] with modifications to simulate nonstandard interactions [24] and accelerator effects, such as the integration of muon decays from positions throughout the decay straight $[31,33]$. The GLoBES simulations assume an experiment with a $1.3 \mathrm{kt}$ far detector at a distance of $2 \mathrm{~km}$ from the end of the storage ring, with $1.6 \times 10^{18}$ useful muon decays. The total appearance signal is 73 events, with a 
LIGHT STERILE NEUTRINO SENSITIVITY AT THE ...

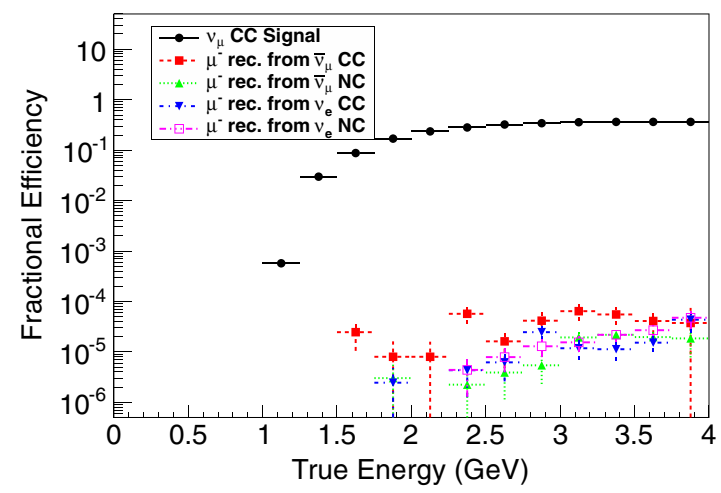

(a)

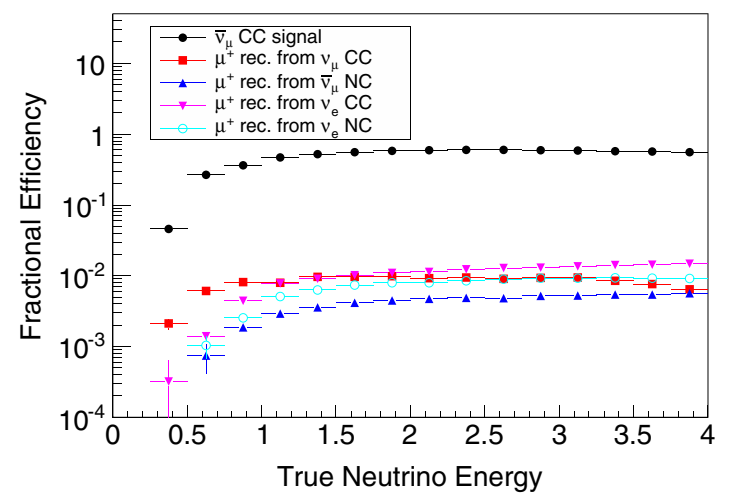

(b)

FIG. 3 (color online). Efficiencies of signals and backgrounds for (a) $\nu_{\mu}$ appearance and (b) $\bar{\nu}_{\mu}$ disappearance for an ironscintillator neutrino detector optimized for the region of interest for nuSTORM. The appearance analysis used a BDT algorithm to define the above curves, while the disappearance analysis used a neural network (MLPBNN) as described in the text.

combined background of 6 events, assuming $\Delta m_{14}^{2}=$ $0.89 \mathrm{eV}^{2}$ and $\theta_{14}=0.15 \mathrm{rad}$.

The sensitivity of a $\nu_{\mu}$ appearance experiment to the presence of sterile neutrinos in a $(3+1)$ model as a function of $\Delta m_{14}^{2}$ and $\sin ^{2} 2 \theta_{e \mu}$ is shown in Fig. 4 assuming the anticipated systematic uncertainties (Table III) and systematic uncertainties inflated to 5\% (signal) and 50\% (background), using a boosted decision tree analysis. This is compared to the $99 \%$ confidence contours from fits generated by Kopp et al. [24] to the combination of LSND, MiniBooNE, and the reactor and gallium disappearance experiments ("Fit to Evid."), and to all available appearance data ("Fit to App.") and to the recent 99\% C.L. contour from the long-baseline ICARUS experiment [34], neglecting matter effects.

Neutrino cross-section uncertainties can be reduced by direct measurements conducted with the beams produced by nuSTORM in both the $\nu_{\mu}$ and $\nu_{e}$ channels. For the appearance experiment, relative systematic uncertainties due to differences in cross sections of neutrino and antineutrino, and electron and muon neutrinos will primarily affect the backgrounds, and therefore are strongly suppressed. The
PHYSICAL REVIEW D 89, 071301(R) (2014)

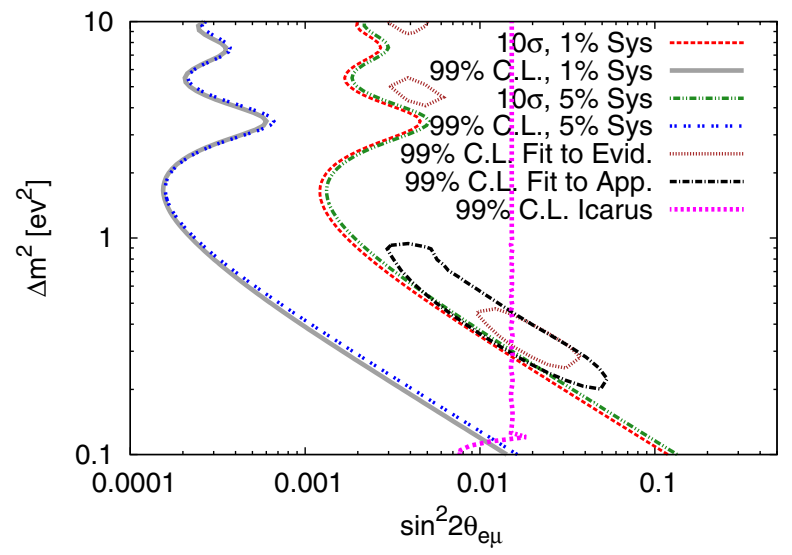

FIG. 4 (color online). Sensitivity of nuSTORM to the $\nu_{e} \rightarrow \nu_{\mu}$ appearance oscillation due to the presence of sterile neutrinos assuming a $(3+1)$ model with anticipated and inflated systematics, compared to $99 \%$ confidence contours from global fits to the evidence for sterile neutrinos and to all available appearance experiments generated by Kopp et al. [24] (filled contours) and limits set by ICARUS [34].

uncertainty in the quasielastic scattering cross section relative to the total cross section will affect the signal and the background equally. Such measurements will greatly contribute to the physics in the neutrino generators used for reference simulations. However, as the appearance search is a rate limited measurement, energy calibration effects such as the known GENIE model uncertainties [35] should not affect the results described here.

The sum of these systematic uncertainties will yield a total $1 \%$ uncertainty to the total normalization of the signal and a $10 \%$ uncertainty to the background. In the absence of any such measurements, an upper limit can be taken from existing experiments, such as MINOS [36]. The convolution of the flux multiplied by the cross section, based on current MINOS data, was used to determine the uncertainties to be $4 \%$ for signal and $40 \%$ for background. For an upper bound to the sensitivity of the described experiment, inflated uncertainties of 5\% and 50\% are considered. The appearance experiment is still sensitive to the presence of a

TABLE III. Systematic uncertainties expected for a shortbaseline muon neutrino appearance experiment based at nuSTORM.

\begin{tabular}{llc}
\hline \hline Uncertainty & \multicolumn{2}{c}{ Expected contribution } \\
Signal & Background \\
\hline Flux & $0.5 \%$ & $0.5 \%$ \\
Cross section & $0.5 \%$ & $5 \%$ \\
Hadronic model & 0 & $8 \%$ \\
Electromagnetic model & $0.5 \%$ & 0 \\
Magnetic field & $0.5 \%$ & $0.5 \%$ \\
Variation in steel thickness & $0.2 \%$ & $0.2 \%$ \\
Total & $1 \%$ & $10 \%$ \\
\hline \hline
\end{tabular}




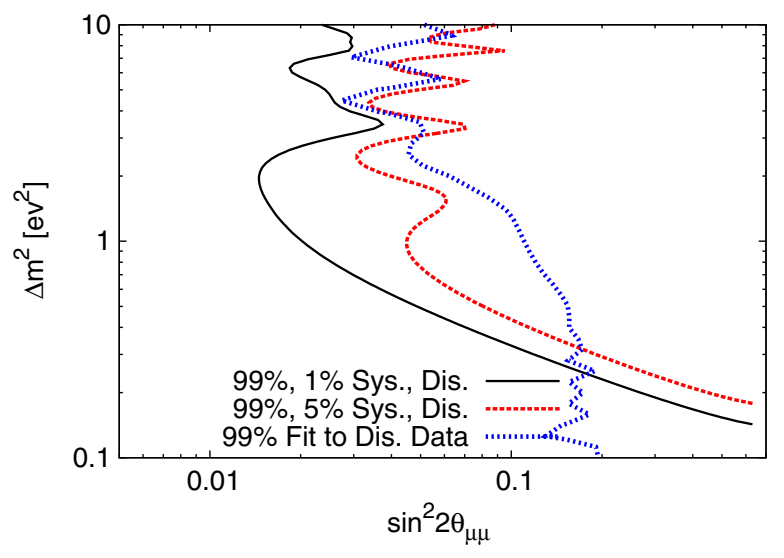

FIG. 5 (color online). Sensitivity of nuSTORM to $\nu_{\mu}$ disappearance oscillations assuming a $(3+1)$ neutrino model. Contours are generated from a $\chi^{2}$ statistic assuming both the anticipated and inflated systematic uncertainties compared to the exclusion contour produced from the fit of $\sin ^{2} 2 \theta_{\mu \mu}$ and $\Delta m^{2}$ to the existing disappearance data (blue dots) [24].

sterile neutrino consistent with the existing evidence at the $10 \sigma$ level, as shown in Fig. 4. Cosmic ray backgrounds were also considered through the application of the CRY software package [37]. With the application of self-vetoing cuts on the fiducial volume to a skin depth of $30 \mathrm{~cm}$, the cosmic ray background is reduced to less than 1 event per year.

A simultaneous and statistically independent $\bar{\nu}_{\mu}$ disappearance measurement will be conducted with the same experimental setup. Sensitivity contours as a function of $\Delta m_{14}^{2}$ and $\sin ^{2} 2 \theta_{\mu \mu}$ are shown in Fig. 5. A near detector is essential to extrapolate the expected neutrino flux at the far detector [38,39]. It is assumed that the systematic uncertainties used in the appearance measurement are the same as those for the disappearance measurement. The $\bar{\nu}_{\mu}$ disappearance measurement is far more sensitive to systematic uncertainties due to the increase of the signal and background acceptance. The exclusion contours set by the nuSTORM disappearance measurement alone shows improvement in the $99 \%$ C.L. bounds over the current global fits as in Fig. 5. The true sensitivity is expected to fall between the pessimistic and optimistic cases, because the inclusion of the flux extrapolation from the 200 Tonne near detector is expected to introduce similar systematic uncertainties while it constrains the spectral uncertainty. The simulation of the near detector required to test this assertion is in progress. An optimization of a $\nu_{e}$ disappearance experiment at a similar muon storage ring facility with idealized detector systems was carried out, demonstrating the near-far extrapolation [40], but the realistic assessment of this channel is still in progress.

The presence of light sterile neutrinos, consistent with the short-baseline neutrino anomalies and from estimates of the effective number of neutrino flavors that arise from fits to cosmological data, would provide evidence for physics beyond the Standard Model and would have far-reaching consequences in neutrino physics and cosmological models of large structure formation. In this article, we have demonstrated that the nuSTORM facility can deliver high purity beams of neutrinos to carry out a $\nu_{e}$ to $\nu_{\mu}$ neutrino oscillation appearance measurement, using an ironscintillator calorimeter detector at a distance of $2 \mathrm{~km}$, with a signal significance of better than $10 \sigma$. The simultaneous use of the $\bar{\nu}_{\mu}$ disappearance channel grants nuSTORM added potential to resolve the current tension between appearance and disappearance measurements and potential to resolve differences between sterile neutrino models. The experimental sensitivity of the appearance channel is largely robust to systematic effects. Therefore, this experiment would be able to provide the definitive test for light sterile neutrinos and resolve a long-standing problem.
[1] A. Aguilar et al. (LSND Collaboration), Phys. Rev. D 64, 112007 (2001).

[2] A. A. Aguilar-Arevalo et al. (MiniBooNE Collaboration), Phys. Rev. Lett. 110, 161801 (2013).

[3] P. Huber, Phys. Rev. C 84, 024617 (2011).

[4] P. Anselmann et al. (GALLEX Collaboration), Phys. Lett. B 342, 440 (1995).

[5] W. Hampel et al. (GALLEX Collaboration), Phys. Lett. B 420, 114 (1998).

[6] J. Abdurashitov, V. Gavrin, S. Girin, V. Gorbachev, T. V. Ibragimova et al., Phys. Rev. Lett. 77, 4708 (1996).

[7] J. Abdurashitov et al. (SAGE Collaboration), Phys. Rev. C 59, 2246 (1999).
[8] J. Abdurashitov, V. Gavrin, S. Girin, V. Gorbachev, P. Gurkina et al. (SAGE Collaboration), Phys. Rev. C 73, 045805 (2006).

[9] K. Mahn et al. (SciBooNE Collaboration, MiniBooNE Collaboration), Phys. Rev. D 85, 032007 (2012).

[10] P. Adamson et al. (MINOS Collaboration), Phys. Rev. Lett. 107, 011802 (2011).

[11] T. Asaka, S. Blanchet, and M. Shaposhnikov, Phys. Lett. B 631, 151 (2005).

[12] P. Ade et al. (Planck Collaboration), arXiv:1303.5076.

[13] T. D. Jacques, L. M. Krauss, and C. Lunardini, Phys. Rev. D 87, 083515 (2013).

[14] S. Gariazzo, C. Giunti, and M. Laveder, J. High Energy Phys. 11 (2013) 211. 
[15] A. Bungau, A. Adelmann, J. Alonso, W. Barletta, R. Barlow et al., Phys. Rev. Lett. 109, 141802 (2012).

[16] K. Abazajian, M. Acero, S. Agarwalla, A. Aguilar-Arevalo, C. Albright et al., arXiv:1204.5379.

[17] A. Donini, M. Gavela, P. Hernandez, and S. Rigolin, Nucl. Phys. B574, 23 (2000).

[18] A. Donini and D. Meloni, Eur. Phys. J. C 22, 179 (2001).

[19] V. Barger, S. Geer, R. Raja, and K. Whisnant, Phys. Rev. D 63, 033002 (2001).

[20] C. Amsler et al. (Particle Data Group), Phys. Lett. B 667, 1 (2008).

[21] A. Hillairet et al. (TWIST Collaboration), Phys. Rev. D 85, 092013 (2012).

[22] P. Kyberd et al. (nuSTORM Collaboration), arXiv:1206.0294.

[23] D. Adey, S. Agarwalla, C. Ankenbrandt, R. Asfandiyarov, J. Back et al., arXiv:1305.1419.

[24] J. Kopp, P. A. N. Machado, M. Maltoni, and T. Schwetz, J. High Energy Phys. 05 (2013) 050.

[25] R. Bayes, A. Laing, F. J. P. Soler, A. C. Villanueva, J. J. Gómez Cadenas, P. Hernández, J. Martín-Albo, and J. Burguet-Castell, Phys. Rev. D 86, 093015 (2012).

[26] C. Andreopoulos et al., Nucl. Instrum. Methods Phys. Res., Sect. A 614, 87 (2010).
[27] J. Apostolakis and D. H. Wright (Geant4 Collaboration), AIP Conf. Proc. 896, 1 (2007).

[28] A. Cervera-Villanueva, J. J. Gomez-Cadenas, and J. A. Hernando, Nucl. Instrum. Methods Phys. Res., Sect. A 534, 180 (2004).

[29] A. Hoecker, J. Stelzer, F. Tegenfeldt, H. Voss, K. Voss et al., Proc. Sci., ACAT2007 (2007) 040.

[30] R. Brun and F. Rademakers, Nucl. Instrum. Methods Phys. Res., Sect. A 389, 81 (1997).

[31] C. Tunnell, Ph.D. thesis, University of Oxford, 2013.

[32] P. Huber, M. Lindner, and W. Winter, Comput. Phys. Commun. 167, 195 (2005).

[33] C. Tunnell, arXiv:1205.6338.

[34] M. Antonello et al., Eur. Phys. J. C 73, 2345 (2013).

[35] P. Coloma, P. Huber, C.-M. Jen, and C. Mariani, arXiv:1311.4506.

[36] P. Adamson et al. (MINOS), Phys. Rev. D 82, 051102 (2010).

[37] C. Hagmann, D. Lange, J. Verbeke, and D. Wright, Lawrence Livermore National Laboratory, Technical Report No. UCRL-TM-229453, 2013.

[38] A. Laing and F. J. P. Soler, AIP Conf. Proc. 981, 166 (2008).

[39] S. Choubey et al. (IDS-NF Collaboration), arXiv:1112.2853.

[40] W. Winter, Phys. Rev. D 85, 113005 (2012). 